\title{
Loss of Signal Transducer and Activator of Transcription 3 Impaired the Osteogenesis of Mesenchymal Progenitor Cells in Vivo and in Vitro
}

\section{ZIJING HUANG}

Sun Yat-Sen University Guanghua School of Stomatology https://orcid.org/0000-0002-7581-6798 Jingyi Feng

Sun Yat-Sen University Guanghua School of Stomatology

\section{Xin Feng}

Sun Yat-Sen University Guanghua School of Stomatology

\section{Laiting Chan}

Sun Yat-Sen University Guanghua School of Stomatology

Jiarui Lu

Sun Yat-Sen University Guanghua School of Stomatology

\section{Lizhen Lei}

Sun Yat-Sen University Guanghua School of Stomatology

\section{Zhuwei Huang}

Sun Yat-Sen University Guanghua School of Stomatology

Xiaolei Zhang ( $\square$ zhangxl35@mail.sysu.edu.cn )

Sun Yat-Sen University Guanghua School of Stomatology https://orcid.org/0000-0003-2410-179X

\section{Research Article}

Keywords: Stat3, bone development, osteogenesis, BMSCs

Posted Date: May 27th, 2021

DOI: https://doi.org/10.21203/rs.3.rs-530012/v1

License: (c) (1) This work is licensed under a Creative Commons Attribution 4.0 International License. Read Full License 


\section{Abstract}

Background: Signal transducer and activator of transcription 3 (Stat3) is a cytoplasmic transcription factor that participates in various biologic processes. Loss of Stat3 causes hyperimmunoglobulin $\mathrm{E}$ syndrome, presenting with skeletal disorders including osteoporosis, recurrent fractures, scoliosis, and craniosynostosis. The objective of this study is to explore the effect and mechanism of Stat3 on osteogenesis of mesenchymal progenitors.

Methods: Stat3 was conditionally knockout (CKO) in mesenchymal progenitors by crossing the pairrelated homeobox gene 1-cre (Prx1-Cre) with Stat3-floxed strain mice. Whole-mount-skeletal staining, histology, and Micro-CT were used to assess the differences between Stat3 CKO and control mice. Further, in vitro experiments were conducted to evaluate the osteogenesis potential of primary isolated bone marrow mesenchymal stem cells (BMSCs) from both control and Stat3 CKO mice. After osteogenic induction for $14 \mathrm{~d}$, alizarin red staining was used to show the calcium deposit, while the western blotting was applied to detect the expression of osteogenic markers.

Results: Compared with the control, Stat3 CKO mice were present with shortened limbs, multiple fractures of long bone, and open calvarial fontanels. The abnormal growth plate structure and reduced collagen fiber were found in Stat3 $\mathrm{CKO}$ limbs. According to micro-CT analysis, the reduced cortical bone thickness and bone volume were found on Stat3 CKO mice. The in vitro osteogenic differentiation of BMSCs was inhibited in Stat3 CKO samples. After osteogenic induction for $14 \mathrm{~d}$, the significantly diminished calcium deposits were found in Stat3 CKO BMSCs. The decreased expression of osteogenic markers (OPN and COL1A1) was observed in Stat3 CKO BMSCs, compared with the control.

Conclusions: Stat3 played a critical role in bone development and osteogenesis. Loss of Stat3 impaired the osteogenesis of mesenchymal progenitors in vivo and in vitro.

\section{Background}

The skeleton development is a complex process involving cell migration, proliferation, and differentiation [1]. The differentiation of mesenchymal progenitors into osteoblasts is critical for both intramembranous and endochondral ossification [2]. Such process requires the participation of multiple signaling pathways. The mutation or abnormal expression of relevant genes can cause serious diseases [2].

Signal transducer and activator of transcription 3 (Stat3) is a cytoplasmic transcription factor and extensively participates in cell proliferation and differentiation [3]. After tyrosine phosphorylation by cytokines and growth factors, Stat3 molecules dimerize and translocate into the nucleus and then regulate gene transcription [4].

Heterozygous Stat3 mutation in humans leads to Job syndrome (also named hyper-IgE syndrome), which is characterized by recurrent multiple organ infection along with skeletal, dental abnormalities [5-7]. The skeletal features of Job syndrome patients comprise recurrent fractures, scoliosis, and cranial- 
maxillofacial abnormalities [7]. Mice with homozygous mutation of the Stat3 gene died during embryonic period, suggesting the essential role of Stat3 for development [8]. Conditional knockout of Stat3 in osteoblast showed an osteoporotic phenotype, while gp130 knockin mice (enhanced activation of Stat3) demonstrated an osteosclerotic phenotype [9]. Moreover, Stat3 disruption in osteoblasts and osteocytes significantly decreased bone strength and reduced load-driven bone formation [10]. These results suggested the important role of Stat3 in osteogenesis of osteoblastic cell lineages. Considering the fact that osteoblastic cells were differentiated from mesenchymal progenitors, the effect of Stat3 on osteogenesis of mesenchymal progenitors deserved an interesting research question to explore.

In the current study, Stat3 was conditional knockout (CKO) in mesenchymal progenitors in vivo by using the pair-related homeobox gene 1-Cre (Prx1-Cre) crossed with Stat3-floxed strain mice [11-13]. The limb and craniofacial bone of Stat3 CKO mice were compared with their littermate control. Bone mesenchymal stem cells were also isolated from the respective mouse lines to assess their osteogenesis potential. The hypothesis of this study is that loss of Stat3 impairs the osteogenesis of mesenchymal progenitors in vivo and in vitro.

\section{Materials And Methods}

\section{Mouse breeding}

The animal work was carried out according to federal guidelines, with the approval by the Animal Ethical and Welfare Committee of Sun Yat-sen University (approval number SYSU-IACUC-2021-000101). Mice were purchased from the Jackson Laboratory (Prx1-Cre, stock no.005584 [11]; Stat3 ${ }^{\mathrm{f} / \mathrm{f}}$, stock no.016923 [13]). To generate mice with Stat3 conditional knockout in limb bud and craniofacial mesenchyme, Stat $3^{f / f}$ were crossed with the Prx1-Cre mice to generate Prx1-Cre; Stat ${ }^{f /+}$ mice. Next, Prx1-Cre; Stat $3^{f /+}$ mice were crossed with Stat $3^{\mathrm{f} / \mathrm{f}}$ mice to generate Prx $1-\mathrm{Cre}$; Stat $3^{\mathrm{f} / \mathrm{f}}$ conditional knockout (CKO) mutant mice. The Cre-negative littermates were taken as the control of Stat3 CKO mutants. Mouse tail genotyping was performed by polymerase chain reaction (PCR) and the primer sequences for PCR were listed in Table 1.

\section{Whole-mount-skeletal staining}

Skeletons from neonatal (P0) mice were processed for Alizarin Red S (sigma A5533) and Alcian Blue (sigma A5268) staining to demonstrate bone and cartilage. The protocol was same as previously described [14]. Pictures were taken by Leica Microsystems (MZ10F). Leica LAS EZ software was used to measure the length of limbs and the width of sagittal sutures.

\section{Histology and immunohistochemical (IHC) staining}

For histology, limbs were fixed in $10 \%$ formalin (Servicebio, Wuhan, China) for $24 \mathrm{~h}$. For 8 -week-old samples, 4 -weeks' decalcification by $10 \%$ tetrasodium EDTA were conducted. After dehydrated by graded ethanol and cleared in xylene, limbs were embedded in paraffin and sectioned at $4 \mu \mathrm{m}$ thickness along the 
long axis. Sections were stained with safranin $\mathrm{O} /$ fast green, masson, hematoxylin and eosin (H\&E) (Servicebio, Wuhan, China). For immunohistochemical staining, the antigen retrieval was performed in a citrate buffer (Beyotime, Shanghai, China). Endogenous peroxidase activities were quenched with $3 \%$ $\mathrm{H}_{2} \mathrm{O}_{2}$ for 10 minutes. Sections were blocked in normal goat serum (NGS) for 1 hour before incubation with primary antibodies overnight at $4^{\circ} \mathrm{C}$. The next day, sections were incubated with biotinylated secondary antibody (Servicebio, Wuhan, China) for 1 hour at room temperature. Then, sections were incubated with diaminobenzidine and counterstained with hematoxylin. Phosphate buffer saline (PBS), instead of primary antibody, was used as the negative control. All sections were mounted in neutral resins and then scanned by AperioAT2 scanner (Leica Microsystems). The quantification of safranin $0 /$ fast green and the Masson staining was carried out by ImageJ. Specifically, in safranin $0 /$ fast green stained sections, the area of proliferative zone and hypertrophic zone was measured respectively, which was then divided by the total area of the growth plate to obtain the relative surface area of the region of interest. In the Masson-stained sections, the blue-stained collagen fiber area was divided by total tissue area to calculate the collagen fraction.

\section{Micro-CT analysis}

The skeletons were scanned and analyzed using a $\mu \mathrm{CT} 50$ system (Scanco, Brüttisellen, Switzerland) with a spatial resolution of $10 \mu \mathrm{m}$. For trabecular bone morphometric analysis of distal femur, the starting slice was taken where the growth plates were fully merged and extended for 100 slices proximally. The morphometric analysis was performed for bone mineral density (BMD) and bone volume per tissue volume (BV/TV; \%) by $\mu \mathrm{CT} 50$ system. The 3D reconstruction of the skeletons and cross-section images were performed by Dataviewer and CTvox (Skyscan, Belgium).

\section{Primary isolation and characterization of BMSCs}

The femur and tibia were dissected from 8-week-old Stat3 CKO mutants and littermate controls. Bone marrow cells from long bones were flushed out by $2 \%$ fetal bovine serum (FBS, Gibco, USA) PBS. Singlecell suspensions from long bone marrow were obtained through $70-\mu \mathrm{m}$ cell mesh and seeded on $100-\mathrm{mm}$ dishes (Jet, Guangzhou, China). Non-adherent cells were removed after 3-days' culture in alpha-MEM (Gibco, USA) supplemented with 20\% FBS (Gibco, USA), $100 \mathrm{U} \cdot \mathrm{mL}^{-1}$ penicillin, and $100 \mu \mathrm{g} \cdot \mathrm{mL}^{-1}$ streptomycin (Gibco, USA), $2 \mathrm{mmol} \cdot \mathrm{L}^{-1}$ I-glutamine (Gibco, USA), $55 \mu \mathrm{mol} \cdot \mathrm{L}^{-1}$ 2-mercaptoethanol (Invitrogen, USA). Cells at passage 2 were employed for the following in vitro experiments.

The mesenchymal stem cell characterization was performed, including (a) the flow cytometry were conducted to show that these cells were positive for CD29, CD44 and were negative for CD34 and CD45 (BD Biosciences, USA), (b) osteoblastic and (c) adipogenic differentiation were applied to show the multilineage differentiation capacity of the primary isolated cells. The antibodies used for flow cytometry were listed in Table 2.

\section{Osteogenic and adipogenic differentiation of BMSCs}


For osteogenic differentiation, the BMSCs were plated in 6-well plates and cultured in osteogenic induction medium containing $10 \mathrm{mM} \beta$-glycerol phosphate (Sigma, USA), and $50 \mu \mathrm{M}$ ascorbic acid (Solarbio, Beijing, China) $10^{-7} \mathrm{M}$ dexamethasone (Sigma, USA). After 7 days' induction, alkaline phosphatase (ALP) expressions were detected by Alkaline Phosphatase Stain kit (Yeasen, Shanghai, China) according to the manufacturer's instructions. Images were taken by Leica Microsystems and ImageJ was applied to quantify the stained areas. After 14-days' osteogenic induction, the calcium deposit was evaluated by Alizarin Red S staining (Cyagen Biosciences, Guangzhou, China). Images were taken by Leica Microsystems, followed by quantification using $10 \%$ cetylpyridinium chloride (CPC) (Sigma, USA) to dissolve the stained calcium deposit. The absorbance value of the dissolved solution at $562 \mathrm{~nm}$ was measured by microplate spectrophotometer (Bio-Tek, UK).

Adipogenic induction medium was provided by Cyagen Biosciences, Inc. (Guangzhou, China). After 12days' induction according to manufacture instruction, the cultured cells were stained with oil red 0 solution (Cyagen, Guangzhou, China), and were observed under light microscope (Leica Microsystems).

\section{Western blot}

Total protein of mouse hindlimbs and BMSCs was extracted using the RIPA lysis buffer (CWBIOTECH, China) containing proteinase and phosphatase inhibitors (CWBIOTECH, China), and then quantified by the BCA method (FudeBio, China). The proteins were separated by SDS-PAGE gel electrophoresis (SmartLifesciences, China) and transferred to a polyvinylidene fluoride (PVDF) membrane (Millipore, USA). After $1 \mathrm{~h}$ blocking by $5 \% \mathrm{BSA}$, membranes were incubated at $4^{\circ} \mathrm{C}$ overnight with relevant primary antibodies. The PVDF membranes were incubated in HRP conjugated secondary antibody for $1 \mathrm{~h}$ at room temperature. Immunoreactivities were detected by a chemiluminescence kit (FudeBio, China). Data were normalized to GAPDH and quantified by ImageJ. The antibodies used in this study were listed in Table 2.

\section{Statistical analysis}

The data were presented as means \pm standard deviation (SD). Student's t-tests were used to compare the difference between Stat3 CKO and the control. The GraphPad Prism 7.0 software were applied for statistical analysis. The significance level was set at $P<0.05$.

\section{Results}

\section{Generation of Stat3 CKO mice}

To investigate the function of Stat3 in skeleton development, Stat3 was deleted in mesenchymal progenitors by use of Prx1-Cre. Fig. 1A demonstrated the mouse genotypes used in this study: Prx1-Cre; $\mathrm{Stat}^{\mathrm{f} / \mathrm{f}}$ (Stat3 $\mathrm{CKO}$ ) and Cre negative littermate control. The deletion of Stat3 was further confirmed by western blot and IHC (Fig. 1B, C). The expression of Stat3 was barely found in bone tissues of Stat3 CKO mice (Fig. 1B, C). 
Conditional deletion of Stat3 in mesenchymal progenitors led to shortened limbs and open fontanels

The Stat3 CKO mice displayed shorten limbs and smaller skull in comparison with the control mice. With aging, the difference of deformed limbs and body size became more obvious between Stat3 CKO mice and their littermate control (Fig. 2A, B). Whole-mount skeletal staining by Alizarin Red and Alcian Blue demonstrated that in Stat3 CKO mice, both forelimbs and hindlimbs were distinctly shorten (Fig. 2C-E) $(P<0.05)$. The abnormalities were found in both proximal long bones (the humerus and femur) and distal long bones (the radius, ulna, tibia and fibula). Multiple fractures were observed in the forelimbs and hindlimbs of Stat3 CKO mice (Fig. 2D). As for cranium, Stat3 CKO mice showed open fontanels and more porous skull when compared with controls (Fig. 2E, G), indicating impaired intramembranous ossification.

\section{Stat3 CKO mice exhibited abnormal growth plate structure and reduced bone volume in limbs}

The neonatal Stat3 CKO mice showed impaired endochondral ossification. The proximal ulna sections of Stat3 CKO mice showed a decrease in the proliferative zones and an increase in the hypertrophic zones (Fig. 3A, B) $(P<0.05)$. Masson's trichrome staining showed that plenty of blue-stained collagen fiber existed in the trabecular bone area of control mice, meanwhile much less collagen was observed on the Stat3 CKO mice (Fig 3C, D) $(P<0.05)$.

The bone parameters were assessed for 8-week-old Stat3 CKO and control mice. The long bones of Stat3 CKO mice were shorter and thicker (Fig 4A and 2D, E). As Fig. 4A showed, Stat3 CKO mice demonstrated irregularly arranged cancellous bone in distal femur, in comparison with the well aligned cancellous bone in littermate control. The cortical bone of Stat3 CKO mice was thinner than the littermate control and some pit-like bone defect can be observed on the surface of cortical bone (Fig. 4A). Micro-CT analysis showed that 8-week-old Stat3 CKO mice was in significant decrease of BV/TV $(P<0.05)$, while the difference in BMD was not significant (Fig. 4B). Histology of 8-week-old Stat3 CKO femur was in accordance with Micro-CT results. The bone marrow volume of femur was also reduced in Stat3 CKO mice, compared with the control (Fig. 4C).

\section{Primary isolation and characterization of BMSCs}

Given that the skeletal abnormalities in Stat3 CKO mice might be owing to defects in bone formation, in vitro experiments were carried out to investigate the cellular mechanism associated with the phenotype. BMSCs were isolated from Stat3 CKO mice and littermate control respectively (Fig. 5A). The primary isolated BMSCs were able to differentiate into adipogenic (Fig. 5B) and osteogenic (Fig. 5C) linage cells, which supported their capacity of multi-lineage differentiation (Fig. 5B, C). In the flow-cytometry, the primary isolated BMSCs were positive for stem cell markers of CD29 and CD44 while negative for hematopoietic markers of CD34 and CD45 (Fig. 5D).

\section{Loss of Stat3 resulted in decreased osteogenesis of BMSCs}

After osteogenic induction for 7d, Stat3 CKO BMSCs showed a pronounced decrease of alkaline phosphatase (ALP) staining intensity, compared with the control $(P<0.05)$ (Fig. 6A). According to the 
alizarin red (ARS) staining, much less calcium deposits were found in Stat3 CKO BMSCs after 14d osteogenic induction, versus in control BMSCs $(P<0.05)$ (Fig. 6B). Quantitative measurements of ALP intensity and Alizarin Red concentration were displayed in Fig. 6C, D. In response to osteogenic induction, Stat3 CKO BMSCs showed a decreased expression of OPN and COL1A1 when compared with the control (Fig. 7A). Further quantitative analyses of protein expression revealed that the decrease of OPN and COL1A1 in Stat3-deficient BMSCs was statistically significant $(P<0.05)$ (Fig. 7B).

\section{Discussion}

In the present study, the severe bone abnormality was observed in Stat3 CKO mice, including shortened limbs, multiple fractures, and reduced bone volume. In vitro study showed that the osteogenic differentiation was reduced in Stat3-deficient BMSCs when compared with the control.

Skeletal development is an orchestrated process comprising intramembranous and endochondral ossification, which both begin with condensation of mesenchymal progenitors [15]. During intramembranous ossification, mesenchymal progenitors directly differentiate into osteoblasts to form mineralized bone. However, during endochondral ossification, mesenchymal progenitors differentiate into chondrocytes and perichondria cells [16]. Then, the chondrocytes undergo proliferation and hypertrophy. The hypertrophic chondrocytes express Indian hedgehog (Ihh) to induce the initial osteoblast differentiation [17]. Growth plate chondrocytes can be replaced by bone and careful coordination of this process are essential for endochondral ossification [16]. In the present study, abnormal growth plate structure was found in Stat3 CKO mice. In the proximal ulna growth plate of Stat3 CKO mice, the proliferative zone was narrower and the hypertrophic zone was wider than the control. The proliferation of chondrocytes contributes greatly to the linear elongation of long bones $[18,19]$. In our study, the shorten limbs in Stat3 CKO mice may result from the decreased proliferative potential of chondrocytes in growth plates. It is known that the hypertrophic chondrocytes express enzymes such as matrix metalloproteinase 13 (MMP13) and undergo apoptosis accompanied with vascular invasion. The apoptosis chondrocytes are replaced by mesenchymal stromal cells that differentiate into osteoblasts to produce bone matrix proteins, the most of which was type 1 collagen $[16,19,20]$. In our study, Stat3 CKO mice showed less collagen fiber in the trabecular bone area when compared with control, especially around the chondroosseous front. This phenomenon might imply that loss of Stat3 can impair the differentiation of mesenchymal stromal cells into osteoblasts, and the ability of osteoblasts to produce bone collagen which is key to form bone matrix.

Stats family has various functions in mammalian development [21, 22]. Among Stats family, Stat3 was believed to be profoundly associated with skeleton homeostasis [23]. In vivo studies have shown that malfunction of Stat3 led to distinguishable bone abnormality in skeletal system $[5,10,24]$. Our previous study demonstrated that overexpression of Stat3 rescued the decreased osteoblast differentiation of Ror2-knockdown mBMSCs, suggesting Stat3 could be the downstream molecule of Ror2 [25]. Ror2 was widely accepted as a transmembrane Wnt receptor in canonical and non-canonical Wnt signaling pathway [26-28]. Mice with conditional knockout of Ror2 in mesenchymal progenitors exhibited shorten 
limbs [25], which was similar to the phenotype of Stat3 CKO mice in the present study. Moreover, the previous study showed a decrease in the width of the proliferative zones and an increase in the width of the hypertrophic zones in the humerus of Ror $2^{-/-}$mice, which was highly consistent with our findings in the proximal ulna of Stat3 CKO mice [29]. This similarity in phenotype of the above studies not only reinforced the importance of Wnt signaling pathway in skeleton development, but also indicated the involvement of Stat3 in Ror2-mediated Wnt signaling.

The in vitro expression of osteogenic markers (OPN and COL1A1) was found diminished in response to Stat3-dificiency. OPN is one of the major non-collagenous proteins that plays key roles in determining bone morphology [30]. Bioinformatics analysis found Stat3 transcription factor binding site (TFBS) in both human and mice OPN gene, indicating that Stat3 might play a role in the transcriptional regulation of OPN gene [6]. The other down-regulated protein in Stat3 CKO BMSCs was COL1A1, which contributed importantly to bone matrix [31]. Fan and colleagues found that in the human hepatic stellate LX-2 cells, COL1A1 might act as a downstream gene in Jak1/Stat3 signaling pathway [32]. The exact interaction between OPN, COL1a1, and Stat3 needs to be further explored. Nevertheless, in our study, Stat3 deficiency resulted in decreased expression of both collagenous (COL1A1) and non-collagenous (OPN) protein in vitro, which in turn, impaired the osteogenic differentiation of BMSCs and less mineralization of the cells was found.

Besides OPN and COL1A1, there were some other genes that could interact with Stat3 according to literatures. Studies have discovered that activated Stat3 could bind to the promoter of several osteogenesis related genes, such as Runx2 [33] and OCN [34]. Xu et a/ reported that icariin could regulate OCN transcription through Stat3 in mBMSCs [34]. More specifically, P-Stat3 interacted with Runx2, forming a complex to bind to the OCN promoter and enhanced mRNA expression of OCN [34]. However, Dalagiorgou et al considered that in mechanically stimulated osteoblasts, P-Stat3 directly bonded to the Runx2 promoter and upregulated mRNA level of Runx2 [33]. The specific transcriptional role of P-Stat3 in osteogenesis might be different under particular circumstances and further research was needed to elucidate the mechanism of Stat3 in BMSC-mediated osteogenesis.

In conclusion, Stat3 was essential for osteogenesis of mesenchymal progenitors in vivo and in vitro. Stat3 CKO mice showed severe skeletal abnormality and Stat3-deficient BMSCs was presented with reduced osteogenic differentiation ability, likely in part by regulating the expression of OPN and COL1A1.

\section{Abbreviations}

Stat3: signal transducer and activator of transcription 3; CKO: conditional knockout; PCR: polymerase chain reaction; IHC: immunohistochemistry; BMSCs: bone marrow mesenchymal stem cells; FBS: fetal bovine serum; BSA: bovine serum albumin; Ror2: receptor tyrosine kinase like orphan receptor 2; OSX: osterix; OPN: osteopontin; COL1A1: collagen I; OCN: osteocalcin; Runx2: runt-related transcription factor-2

\section{Declarations}




\section{Ethics approval and consent to participate}

Mice were used according to federal guidelines and as approved by the Animal Ethical and Welfare Committee of Sun Yat-sen University (Approval Number SYSU-IACUC-2021-000101).

\section{Consent for publication}

Not applicable.

\section{Availability of data and materials}

All datasets used and/or analyzed during the current study are available from the corresponding author on reasonable request.

\section{Competing interests}

The authors declare that they have no competing interests.

\section{Founding}

This work was supported by the National Natural Science Foundation of China (Grant No. 81470731).

\section{Authors' Contributions}

ZJH, JYF and XLZ designed the experiments; ZJH, JYF, XF, LTC, JRL, LZL and ZWH performed experiments; ZJH and JYF analyzed the data; ZJH wrote the manuscript; XLZ participated in critical revision of the paper. All authors read and approved the final manuscript. All authors discussed the results and approved the final manuscript.

\section{Acknowledgements}

Not applicable.

\section{References}

1. Karsenty G. The complexities of skeletal biology. Nature. 2003; 423(6937):316-8.

2. Long F. Building strong bones: molecular regulation of the osteoblast lineage. Nature Reviews Molecular Cell Biology. 2011; 13(1):27-38.

3. Liu Y, Liao S, Bennett S, Tang H, Song D, Wood D, et al. STAT3 and its targeting inhibitors in osteosarcoma. Cell Prolif. 2020:e12974.

4. Yu H, Kortylewski M, Pardoll D. Crosstalk between cancer and immune cells: role of STAT3 in the tumour microenvironment. Nat Rev Immunol. 2007; 7(1):41-51.

5. Holland SM, DeLeo FR, Elloumi HZ, Hsu AP, Uzel G, Brodsky N, et al. STAT3 mutations in the hyper-IgE syndrome. N Engl J Med. 2007; 357(16):1608-19. 
6. Goel S, Sahu S, Minz RW, Singh S, Suri D, Oh YM, et al. STAT3-Mediated Transcriptional Regulation of Osteopontin in STAT3 Loss-of-Function Related Hyper IgE Syndrome. Front Immunol. 2018; 9:1080.

7. Al-Shaikhly T, Ochs HD. Hyper IgE syndromes: clinical and molecular characteristics. Immunol Cell Biol. 2019; 97(4):368-379.

8. Takeda K, Noguchi K, Shi W, Tanaka T, Matsumoto M, Yoshida N, et al. Targeted disruption of the mouse Stat3 gene leads to early embryonic lethality. Proc Natl Acad Sci U S A. 1997; 94(8):3801-4.

9. Itoh S, Udagawa N, Takahashi N, Yoshitake F, Narita H, Ebisu S, et al. A critical role for interleukin-6 family-mediated Stat3 activation in osteoblast differentiation and bone formation. Bone. 2006; 39(3):505-12.

10. Zhou H, Newnum AB, Martin JR, Li P, Nelson MT, Moh A, et al. Osteoblast/osteocyte-specific inactivation of Stat3 decreases load-driven bone formation and accumulates reactive oxygen species. Bone. 2011; 49(3):404-11.

11. Logan M, Martin JF, Nagy A, Lobe C, Olson EN, Tabin CJ. Expression of Cre recombinase in the developing mouse limb bud driven by aPrxl enhancer. genesis. 2002; 33(2):77-80.

12. Kim H, Kim M, Im SK, Fang S. Mouse Cre-LoxP system: general principles to determine tissue-specific roles of target genes. Lab Anim Res. 2018; 34(4):147-159.

13. Moh A, Iwamoto Y, Chai GX, Zhang SS, Kano A, Yang DD, et al. Role of STAT3 in liver regeneration: survival, DNA synthesis, inflammatory reaction and liver mass recovery. Lab Invest. 2007; 87(10):1018-28.

14. Rigueur D, Lyons KM. Whole-mount skeletal staining. Methods Mol Biol. 2014; 1130:113-121.

15. Hall BK, Miyake T. All for one and one for all: condensations and the initiation of skeletal development. Bioessays. 2000; 22(2):138-47.

16. Kronenberg HM. Developmental regulation of the growth plate. Nature. 2003; 423(6937):332-6.

17. Long F, Chung UI, Ohba S, McMahon J, Kronenberg HM, McMahon AP. Ihh signaling is directly required for the osteoblast lineage in the endochondral skeleton. Development. 2004; 131(6):130918.

18. Deng C, Wynshaw-Boris A, Zhou F, Kuo A, Leder P. Fibroblast growth factor receptor 3 is a negative regulator of bone growth. Cell. 1996; 84(6):911-21.

19. Long F, Ornitz DM. Development of the endochondral skeleton. Cold Spring Harb Perspect Biol. 2013; 5(1):a008334.

20. Aghajanian P, Mohan S. The art of building bone: emerging role of chondrocyte-to-osteoblast transdifferentiation in endochondral ossification. Bone Res. 2018; 6:19.

21. Darnell JE. STATs and gene regulation. Science. 1997; 277(5332):1630-5.

22. Abroun S, Saki N, Ahmadvand M, Asghari F, Salari F, Rahim F. STATs: An Old Story, Yet Mesmerizing. Cell J. 2015; 17(3):395-411.

23. Li J. JAK-STAT and bone metabolism. JAKSTAT. 2013; 2(3):e23930. 
24. Hall MD, Murray CA, Valdez MJ, Perantoni AO. Mesoderm-specific Stat3 deletion affects expression of Sox9 yielding Sox9-dependent phenotypes. PLoS Genet. 2017; 13(2):e1006610.

25. Lei L, Huang Z, Feng J, Huang Z, Tao Y, Hu X, et al. Loss of receptor tyrosine kinase-like orphan receptor 2 impairs the osteogenesis of mBMSCs by inhibiting signal transducer and activator of transcription 3. Stem Cell Res Ther. 2020; 11(1):137.

26. Oishi I, Suzuki H, Onishi N, Takada R, Kani S, Ohkawara B, et al. The receptor tyrosine kinase Ror2 is involved in non-canonical Wnt5a/JNK signalling pathway. Genes to Cells. 2003; 8(7):645-654.

27. Billiard J, Way DS, Seestaller-Wehr LM, Moran RA, Mangine A, Bodine PV. The orphan receptor tyrosine kinase Ror2 modulates canonical Wnt signaling in osteoblastic cells. Mol Endocrinol. 2005; 19(1):90-101.

28. Ling L, Nurcombe V, Cool SM. Wnt signaling controls the fate of mesenchymal stem cells. Gene. 2009; 433(1-2):1-7.

29. DeChiara TM, Kimble RB, Poueymirou WT, Rojas J, Masiakowski P, Valenzuela DM et al. Ror2, encoding a receptor-like tyrosine kinase, is required for cartilage and growth plate development. . Nat Genet. 2000; 24:271-274.

30. Bailey S, Karsenty G, Gundberg C, and Vashishth D. Osteocalcin and osteopontin influence bone morphology and mechanical properties. Ann N Y Acad Sci. 2017; 1409(1):79-84.

31. Clarke B. Normal bone anatomy and physiology. Clin J Am Soc Nephrol. 2008; 3 Suppl 3:S131-9.

32. Fan T, Ge M, Guo Z, He H, Zhang N, Li Y, et al. Discovery of 90-Substituted Palmatine Derivatives as a New Class of anti-COL1A1 Agents via Repressing TGF-beta1/Smads and JAK1/STAT3 Pathways. Molecules. 2020; 25(4).

33. Dalagiorgou G, Piperi C, Adamopoulos C, Georgopoulou U, Gargalionis AN, Spyropoulou A, et al. Mechanosensor polycystin-1 potentiates differentiation of human osteoblastic cells by upregulating Runx2 expression via induction of JAK2/STAT3 signaling axis. Cell Mol Life Sci. 2017; 74(5):921936.

34. Xu H, Zhou S, Qu R, Yang Y, Gong X, Hong Y, et al. Icariin prevents oestrogen deficiency-induced alveolar bone loss through promoting osteogenesis via STAT3. Cell Prolif. 2020; 53(2):e12743.

\section{Tables}

Table 1 Primers for genotyping

\begin{tabular}{|lll|}
\hline Gene & Forward & Reverse \\
\hline Prx1-cre & GCGGTCTGGCAGTAAAAACTATC & GTGAAACAGCATTGCTGTCACTT \\
\hline Stat3 & TTGACCTGTGCTCCTACAAAAA & CCCTAGATTAGGCCAGCACA \\
\hline
\end{tabular}


Table 2 Antibodies for flow cytometry, western blot and immunochemistry

\begin{tabular}{|lll|}
\hline Antibody raised against & Dilution & Source (cat. NO) \\
\hline Flow cytometry antibody & & \\
\hline PE Rat IgG2b, Klsotype Control & $1: 200(F)$ & BD (553989) \\
\hline FITC Rat IgG2b, klsotype Control & $1: 200(F)$ & BD (554688) \\
\hline PE Hamster Anti-Mouse CD29 & $1: 200(F)$ & BD (562801) \\
\hline PE Rat Anti-Mouse CD44 & $1: 200(F)$ & BD (553134) \\
\hline FITC Rat Anti-Mouse CD34 & $1: 200(F)$ & BD (560238) \\
\hline FITC Rat Anti-Mouse CD45 & $1: 200(F)$ & BD (553079) \\
\hline Primary antibody & & \\
\hline Stat3 mouse mAb & $1: 500($ IHC) & CST (9139) \\
\hline PStat3 mouse mAb & $1: 1000(\mathrm{WB})$ & CST (9139) \\
\hline OPN rabbit pAb & $1: 1500(\mathrm{WB})$ & CST (4113) \\
\hline COL1A1 rabbit pAb & $1: 1000(\mathrm{WB})$ & ZEN BIO (380437) \\
\hline GAPDH rabbit mAb & $1: 1000(\mathrm{WB})$ & Abclonal (A1352) \\
\hline Secondary antibody & $1: 1000(\mathrm{WB})$ & CST (5174) \\
\hline Anti-mouse IgG HRP-linked Ab & $1: 4000(\mathrm{WB})$ & CST (7076) \\
\hline Anti-rabbit IgG HRP-linked Ab & $1: 4000(\mathrm{WB})$ & CST (7074) \\
\hline
\end{tabular}

\section{Figures}

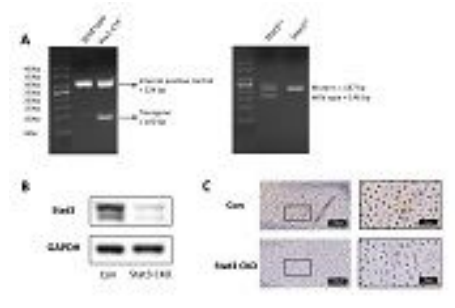

Figure 1 
In vivo deletion of Stat3 in mesenchymal progenitors. A. Genotyping of Stat3f/f; Prx1-Cre mice and Stat3f/+ mice tail DNA. DNA ladder: $50 \mathrm{bp}$. B. Protein were extracted from P0 forelimbs. Western blot analysis revealed significant decrease of Stat3 protein expression in Stat3 CKO compared with control. C. Immunohistochemistry (IHC) for Stat3 in E14.5 ulna sections showed the loss of Stat3 expression in Stat3 CKO. The boxed regions are enlarged in the right panel. Arrows indicate Stat3-positive cells.
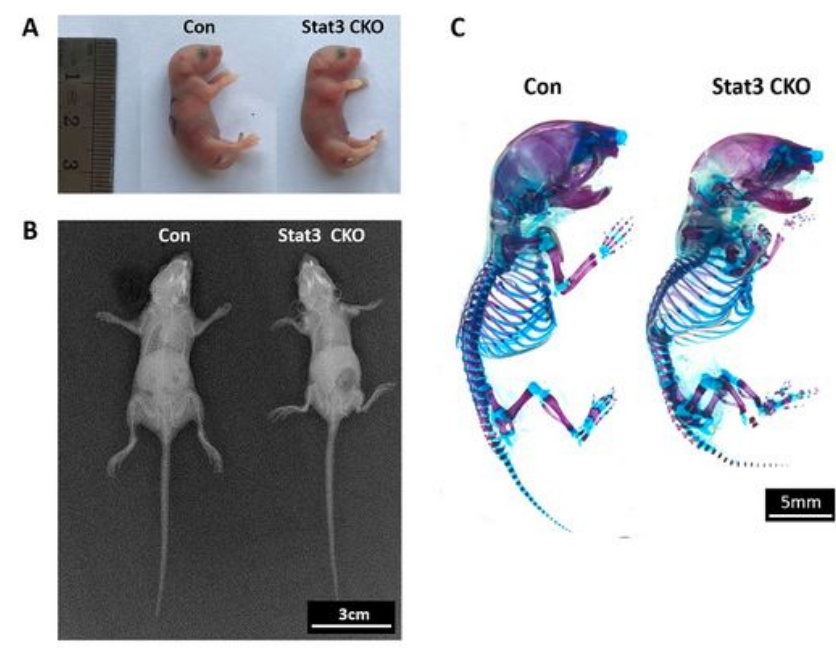
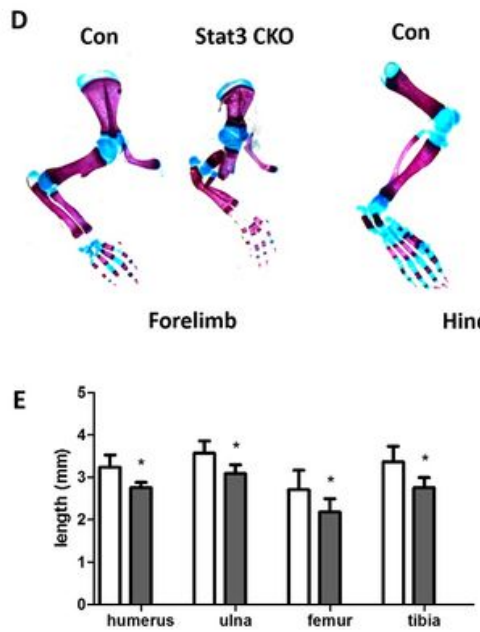
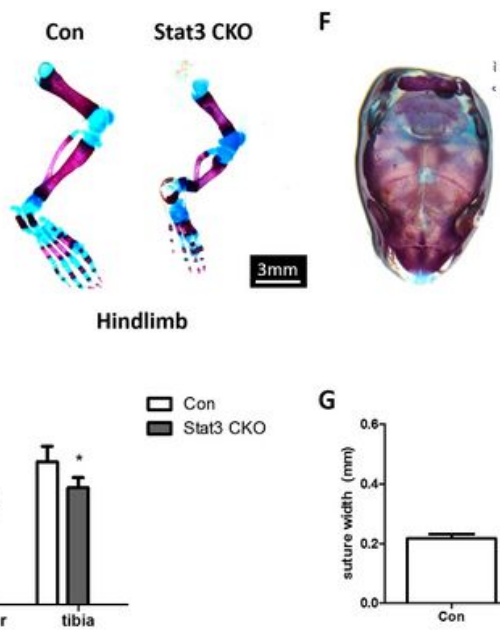

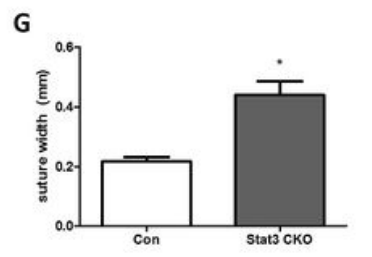

Figure 2

Loss of Stat3 resulted in skeletal abnormalities. A, B. Ex vivo observation of control and Stat3 CKO mice at PO and 3w. C. Alizarin red/alcian blue staining of skeletal preparations of PO control and Stat3 CKO mice. D, E. Multiple fractures were observed in Stat3 CKO mice. Compared with the littermate control, the limbs were significantly shortened. F, G. Alizarin red/alcian blue from P0 samples showed dysplasia in Stat3 CKO calvaria. Compared with the littermate control, the width of sagittal suture was significantly wider in Stat3 CKO mice. Quantitative data were obtained using the Image J software. $n=3, * P<0.05$ vs Con. 

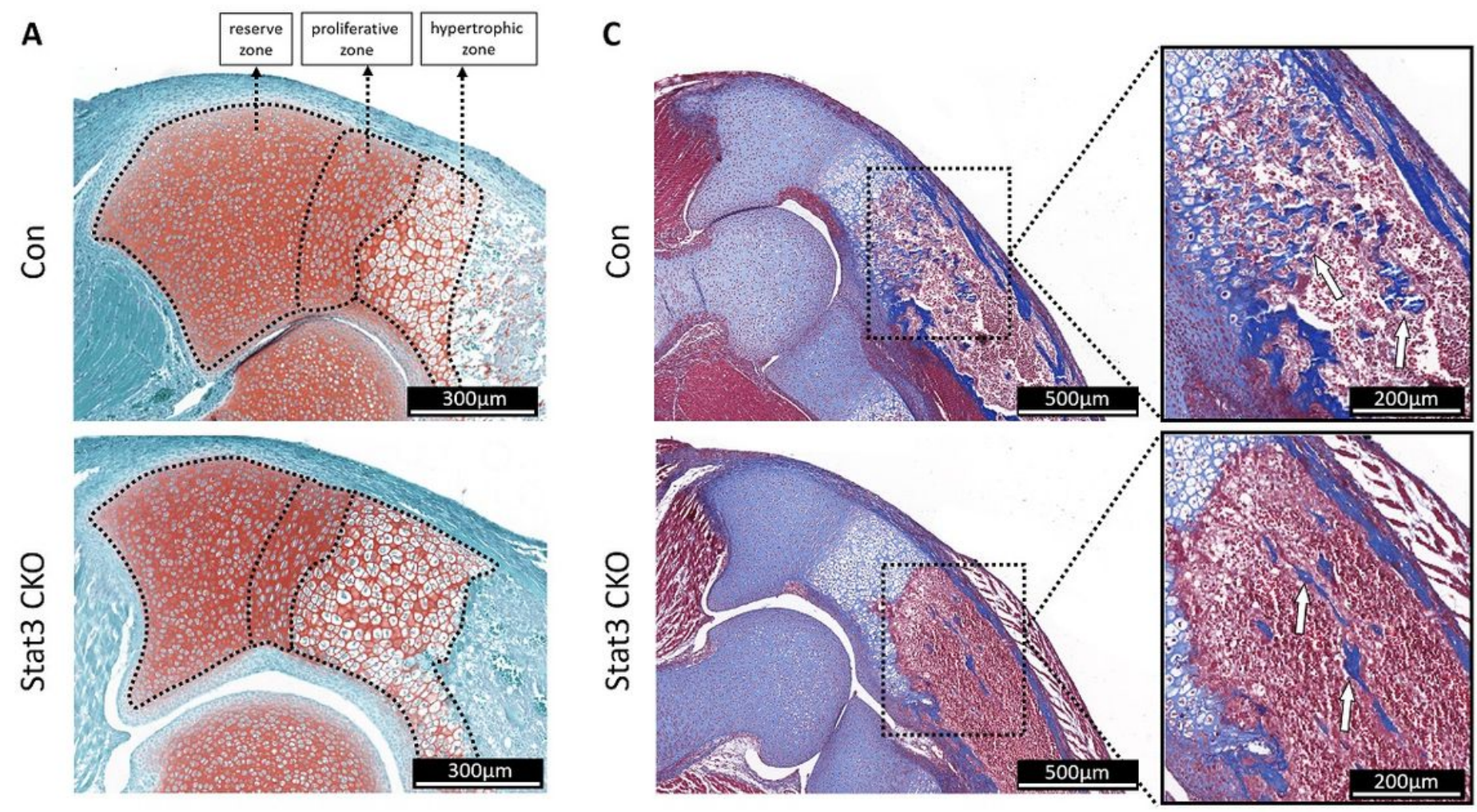

B

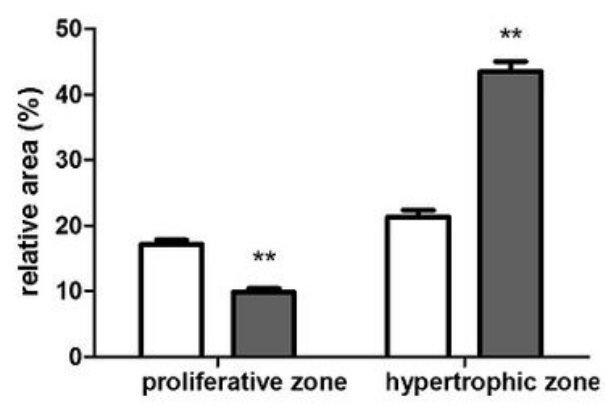

므 Con

Stat3 CKO
D

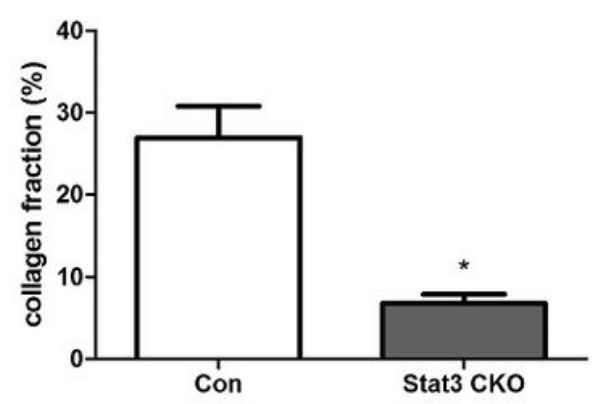

\section{Figure 3}

The impaired endochondral ossification was found on neonatal Stat3 CKO mice. A, B. Safranin O/fast green staining of proximal ulna sections showed a decrease in the proliferative zone and an increase in the hypertrophic zone in Stat3 CKO samples when compared with the control. C, D. The masson staining showed less blue-stained collagen fibers in the trabecular bone region of Stat3 CKO mice. The boxed region was enlarged in the right panel, white arrows pointing to stained collagen fibers. $n=3,{ }^{*} P<0.05$, $\star \star P<0.01$ vs Con. 
A
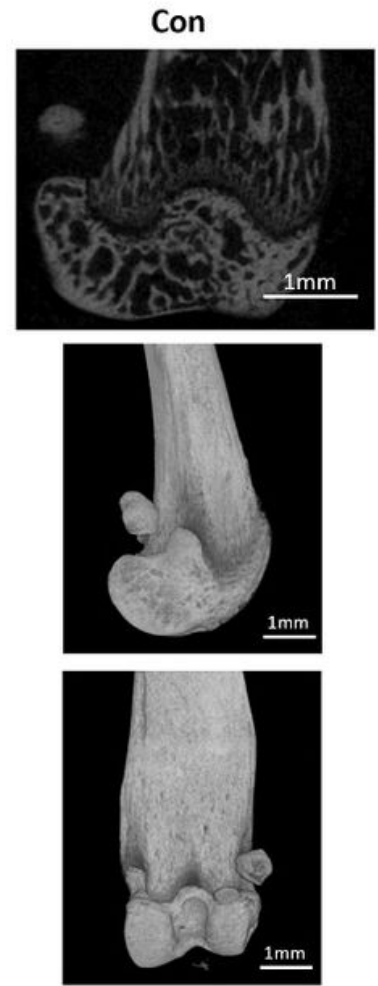

Stat3 CKO
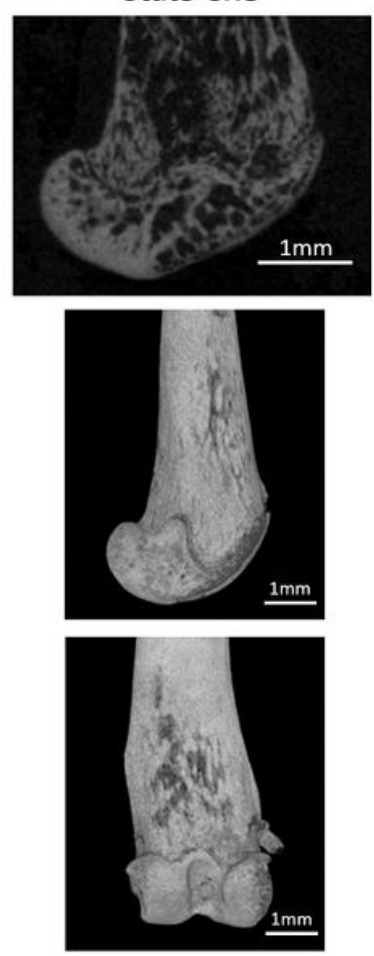

B
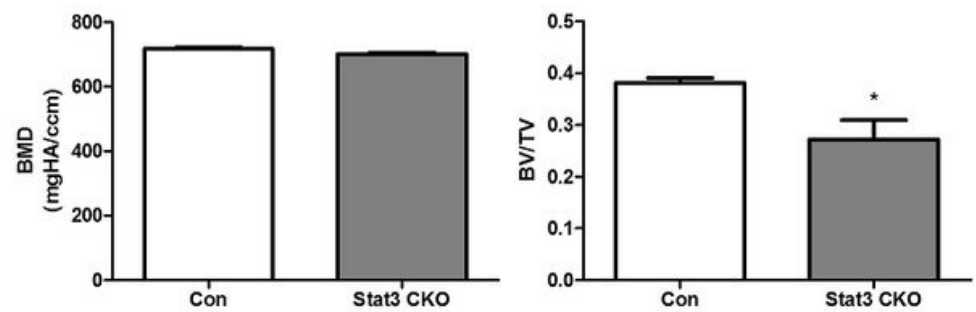

C

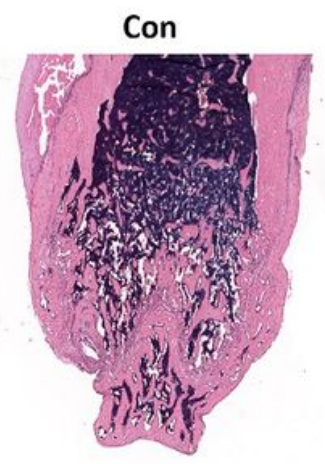

Stat3 CKO

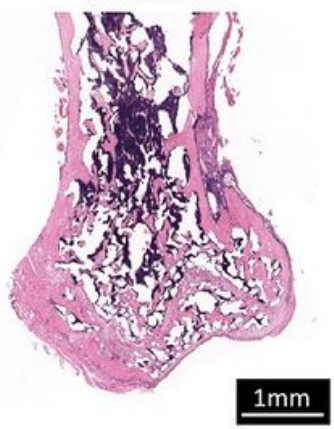

Figure 4

Defects in cancellous and cortical bone in Stat3 CKO mice of 8-week-old. A, B. Micro-CT analysis of femurs from 8-week-old control and Stat3 CKO mice. Irregular cancellous and thinner cortical bone were observed in Stat3 CKO mice (A). Quantification of micro-CT data showed a significantly reduced BV/TV at femur growth plate $(n=3, * P<0.05$ vs Con). BMD: bone mineral density; BV/TV: bone volume/tissue volume. C. H\&E-stained longitudinal sections of femurs from 8-week-old control and Stat3 CKO mice.

A

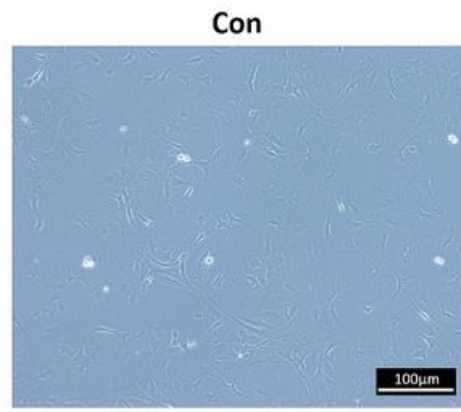

B

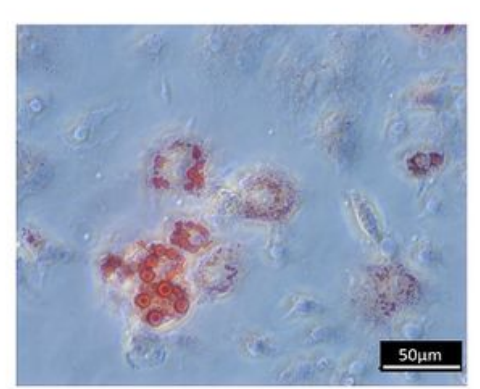

Stat3 CKO

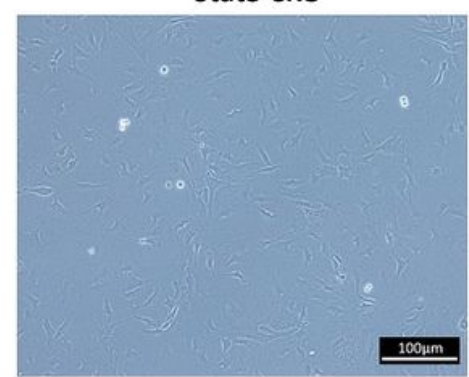

C

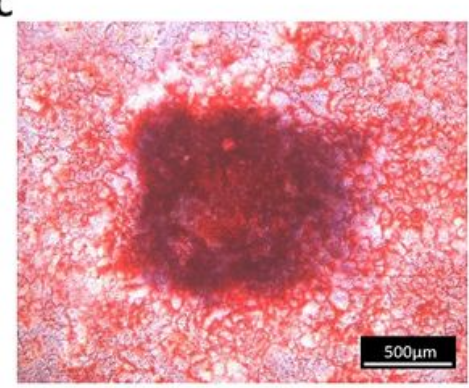

D
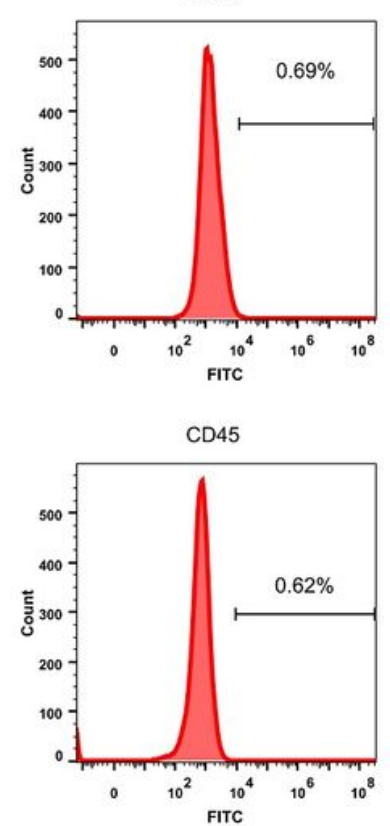

CD44

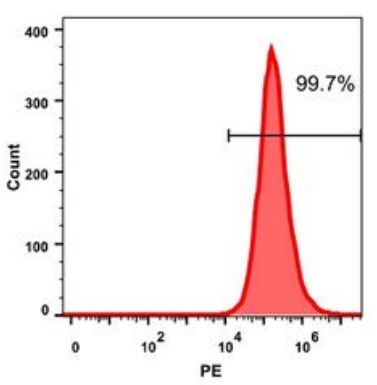

CD29

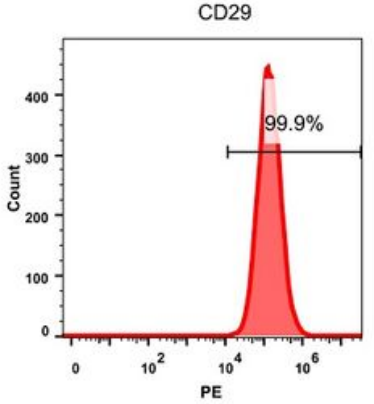


Figure 5

Isolation and characterization of bone marrow mesenchymal stem cells (BMSCs). A. Primary isolation of bone marrow mesenchymal stem cells (BMSCs) (5-week-old mouse, passage 2). B. Oil red 0 staining were performed after adipogenic induction for $12 \mathrm{~d}$. C. Alizarin red staining were performed after osteogenic induction for 14d. D. Flow cytometry analysis of cell surface markers of CD29, CD44, CD34, and CD45 in primary isolated BMSCs.

A
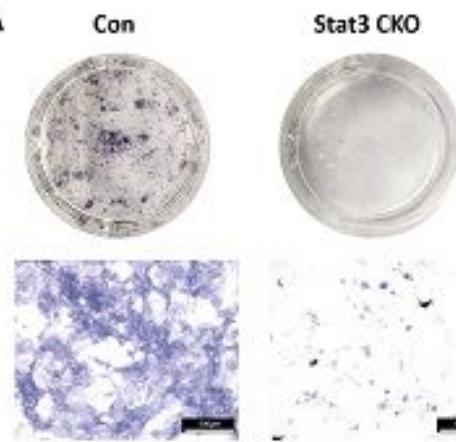

B
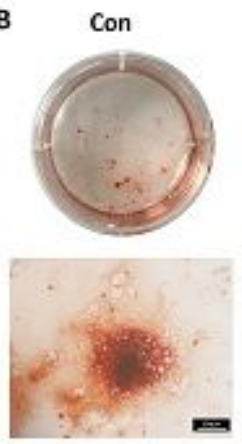

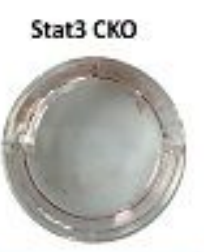

C

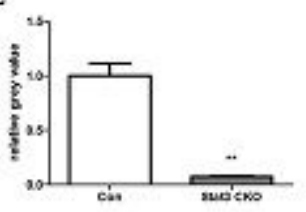

D

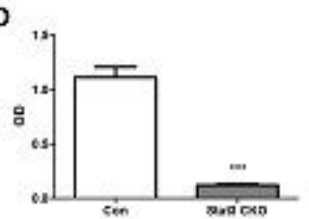

Figure 6

The decreased ALP expression and mineralization were found on Stat3 CKO BMSCs. The alkaline phosphatase (ALP) staining was performed at 7d of osteogenic induction (A). The alizarin red staining (ARS) was done at $14 \mathrm{~d}$ of osteogenic induction (B). C. Quantitative data of ALP staining were obtained by Image J software D. Alizarin Red concentrations were determined by a quantitative destaining procedure using cetylpyridinium chloride (CPC). $n=3,{ }^{*} P<0.01, * \star * P<0.001$ vs Con.

A

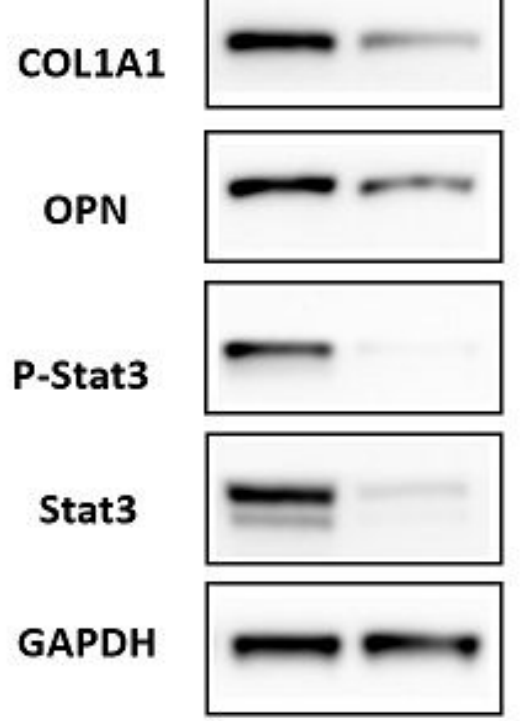

Con Stat3 CKO
B

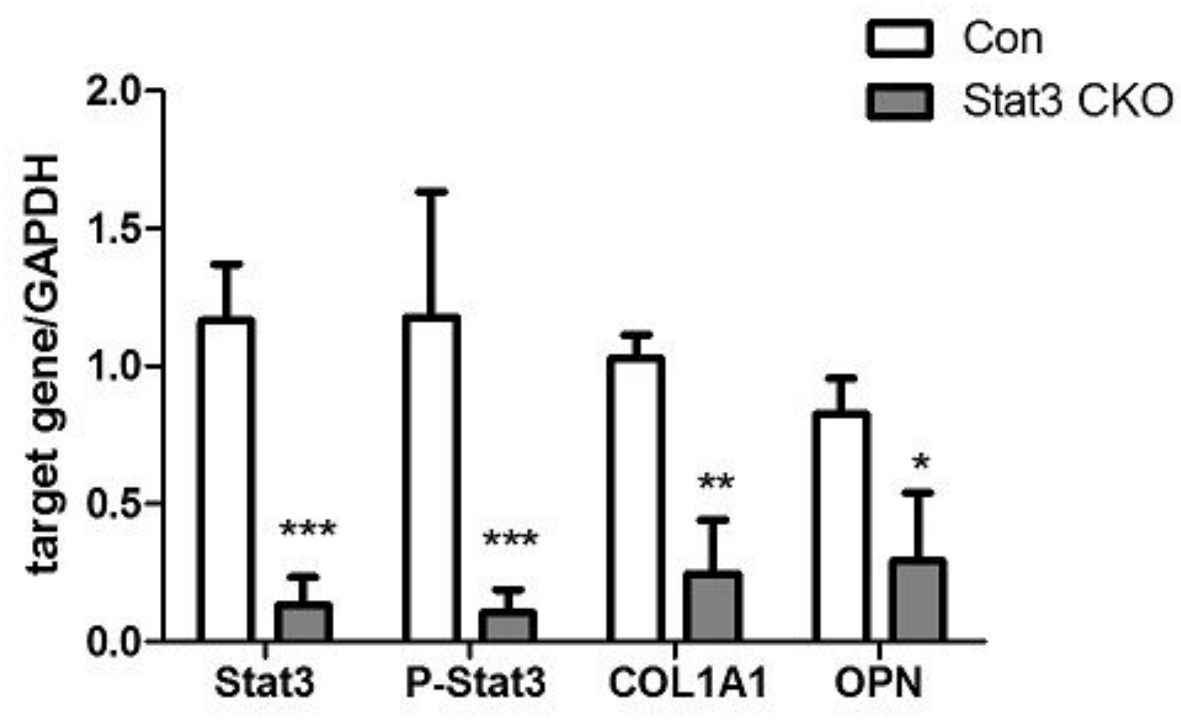




\section{Figure 7}

After osteogenic induction for 14 days, Stat3 CKO BMSCs expressed less osteogenic makers when compared to the control. A. BMSCs expressions of osteogenic markers were assessed by western blotting. B. Quantitative analysis of Stat3, P-Stat3 and osteogenic markers (COL1A1 and OPN) expression levels. $n=3 .{ }^{*}<<0.05, * \star P<0.01, * \star * P<0.001$ vs Con. 